\title{
Fast and Accurate Frequency Estimation for Renewable Energy Systems Using Maximum Decay Sidelobes Windows
}

\author{
Dariusz Kania $^{1}$, Józef Borkowski ${ }^{1}$, Janusz Mroczka ${ }^{1}$ \\ ${ }^{1}$ Chair of Electronic and Photonic Metrology, Wrocław University of Technology, Poland \\ B. Prusa 53/55,50-317 Wrocław, Poland \\ e-mail: Dariusz.Kania@pwr.wroc.pl, Jozef.Borkowski@pwr.wroc.pl, Janusz.Mroczka@pwr.wroc.pl
}

\begin{abstract}
Fast and accurate signal frequency estimation has great importance i.a. in renewable energy systems and power control systems. The energy produced by these systems shall fulfill quality requirements as defined in the respective directives and standards. More accurate and faster grid signal frequency estimation (which can be used to control the inverter) can improve the energy quality. This article presents an overview of methods for spectrum interpolation and signal frequency estimation and a generalized method for very accurate and fast frequency grid estimation by using the Fast Fourier Transform procedure and maximum decay sidelobes windows. An important feature of this algorithm is the elimination of the impact of the conjugate component on the estimation result. The article shows the statistical properties of the frequency estimator as well as the effect of noise for the estimation. The results of the simulation show that the algorithm can be successfully used for a fast and accurate estimation of the grid signal frequency i.a. in renewable energy systems and power control systems. The accuracy of the frequency estimation is in the order of $5 \cdot 10^{-11} \mathrm{~Hz}$ for a $5 \mathrm{~ms}$ measurement window. Using different windows from the discussed family allows to adjust to the actual requirements of estimation.
\end{abstract}

\section{Keywords}

grid frequency estimation, control of power, renewable energy, interpolated DFT, maximum decay sidelobes windows.

\section{Introduction}

Obtaining electrical energy from renewable energy sources has become very popular in recent years in many countries around the world [1]. Renewable energy systems and power control systems are composed of several blocks, in which frequency estimation has great importance. Estimation of a sinusoidal frequency is also very important in many other areas of science and technology (e.g. medicine [2], electronics [3]). In the power industry the estimation must be accurate because of the need for the assessment of the power quality. When the quality is lower, the financial losses are greater [4] and the energy system operates with lower overall efficiency. Low quality might be caused by an external source (e.g. a photovoltaic system) connected to the grid that has no appropriate controls. In other renewable energy systems accurate and fast estimation of the grid signal parameters is also essential for proper production energy in different weather conditions [5]-[10]. Accurate and prompt detection of these parameters (especially frequency) is necessary for the generation of appropriate signal and for the compliance with certain quality standards of energy production [11]-[13].

The IEC 61727-2002 standard requires that, under certain conditions, a photovoltaic system has to stop power output no later than after $50 \mathrm{~ms}$ (after 2.5 cycles of a grid signal that is characterized by the frequency of 50 $\mathrm{Hz}$ ) [13]. The next standard, IEEE Std 929-2000, requires a system turn-off after two cycles of $60 \mathrm{~Hz}$ grid signal [14].

A classical spectral method of analysis is often used to estimate the sinusoidal signal frequency. Such methods use time windows and the Fast Fourier Transform (FFT) algorithm [15]-[17]. Additionally, by using the methods of spectrum interpolation (IpDFT) a significant improvement in the estimation accuracy is obtained [18][34]. An important group of methods are the methods of DFT interpolation, which account, in their equations, for the phenomenon of spectral leakage. These methods include the group of multi-point weighted interpolations of DFT methods (MWIDFT) [19], [20], [24] and a linear interpolation of the DFT (LIDFT) [25]-[27], based on an approximation of the unit circle by a polygon.

This paper presents a generalization of the interpolation method MWDIFT [28] for maximum decay sidelobes windows at an arbitrary order, which allows to estimate the frequency of the fundamental sinusoidal component of a multi-frequency signal in a short measurement time (measurement of 0.5-2.5 measured signal cycles). This model is adequate for $50 / 60 \mathrm{~Hz}$ power signal which has harmonics and for time measurement requirements in renewable energy systems. 


\section{Frequency estimation using maximum decay sidelobes windows}

A multi-frequency signal can be described in the time domain as follows:

$$
x(t)=\sum_{i=1}^{M} A_{i} \sin \left(2 \pi f_{i} t+\varphi_{i}\right)
$$

where

$A_{i}$ - amplitude of the $i$-th component,

$f_{i}$ - frequency of the $i$-th component,

$\varphi_{i}$ - phase angle of the $i$-th component.

After a process of sampling (with a frequency of $\left.f_{\mathrm{s}}=1 / T\right)$, the $x(t)$ signal is represented by a series of discrete $N$ values (samples) $x_{n}=x(n T)$, where $n=0, \ldots, N-1$. The frequency $f_{i}$ in (1) can be normalized with respect to the measurement time $N T$ by $\lambda_{\mathrm{i}}=f_{i} N T$ frequency, and, in general, $\lambda=f N T[\mathrm{~Hz} / \mathrm{Hz}]$ for any $f$. The unit of $\lambda$ is marked as a bin. The frequency $\lambda$ is also referred to as CiR (Cycles in Range) [28]. It emphasizes that $\lambda$ specifies the number of sinusoidal component cycles in the time window that comprises $N$ signal samples.

The cosine windows family is an important group of time windows. The time window samples $w_{n}$ of these windows are defined in [20], [29]-[31] as follows:

$$
w_{n}=\sum_{h=0}^{H-1}(-1)^{h} a_{h} \cos \frac{2 \pi n h}{N}, n=0, \ldots, N-1
$$

The maximum decay sidelobes windows (I class RifeVincent windows, binomial coefficient windows) are among these windows. They were defined for the first time by Rife and Vincent in 1970 [29], and have maximum decay amplitudes of sidelobes from all cosine window families defined by (2). The relations for the maximum decay sidelobes window coefficients are [31]:

$$
a_{0}=\frac{C_{2 H-2}^{H-1}}{2^{2 H-2}} ; a_{h}=\frac{C_{2 H-2}^{H-h-1}}{2^{2 H-3}}, h=1, \ldots, H-1
$$

where

$$
C_{m}^{p}=\left(\begin{array}{c}
m \\
p
\end{array}\right)=\frac{m !}{(m-p) ! p !}
$$

and $H$ is the window order that equals the number of coefficients $a_{h}$. Special cases are a rectangular window $(H=1)$ and a Hanning (Hann) window $(H=2)$.

The spectrum of I class Rife-Vincent windows can be approximated for $H>1, \lambda \geqslant N, N \geqslant 1$ by [20], [30]:

$$
W(\lambda)=\frac{D(\lambda)}{P(\lambda)}
$$

where

$$
D(\lambda)=\frac{N(2 H-2) !}{2^{2 H-2}} \sin (\pi \lambda) e^{-j \pi \lambda}
$$

$$
P(\lambda)=\lambda \prod_{h=1}^{H}\left(h^{2}-\lambda^{2}\right)=\frac{\prod_{0}^{H}\left(h^{2}-\lambda^{2}\right)}{(-\lambda)(H-\lambda)(H+\lambda)}
$$

In the paper, this property of the group of multi-point weighted interpolations of DFT methods has been used to estimate the frequency of the sinusoidal component. The value of estimated frequency is low (grid frequency) and the impact of the conjugate component is important.

The MWIDFT methods that consider the impact of long-range leakage are still being developed [19], [20], [32], [33]. The method from [28] allows to determine the normalized frequency $\lambda_{1}(9)$, which considers the effect of a conjugate component with a frequency of $-\lambda_{1}$ (Fig. 1). This method uses the Hanning time window and it is based on the relevant three consecutive points of the spectrum $X(\lambda)$ defined as:

$$
X(\lambda)=\sum_{n=0}^{N-1} x_{n} w_{n} e^{-j 2 \pi n \lambda / N}
$$

for $\lambda=k-1, k, k+1$ (values $X_{k-1}, X_{k}, X_{k+1}$ ) around the main lobe.

$$
\lambda_{1}= \pm \sqrt{\frac{\Pi_{1}}{\Pi_{2}}}
$$

where

$$
\begin{gathered}
\Pi_{1}=\left|\begin{array}{ccc}
6 & 3 & X_{k-1}-X_{k} \\
-k^{2}-4 & 2 k & X_{k} \\
6 & -3 & X_{k+1}-X_{k}
\end{array}\right| \\
\Pi_{2}=\left|\begin{array}{ccc}
1 & -3 & X_{k-1} \\
1 & 0 & X_{k} \\
1 & 3 & X_{k+1}
\end{array}\right|
\end{gathered}
$$

$$
\begin{array}{ll}
\text { a) } w_{n} \text { - rectangular window } & \text { b) } w_{n} \text { - Hanning window }
\end{array}
$$

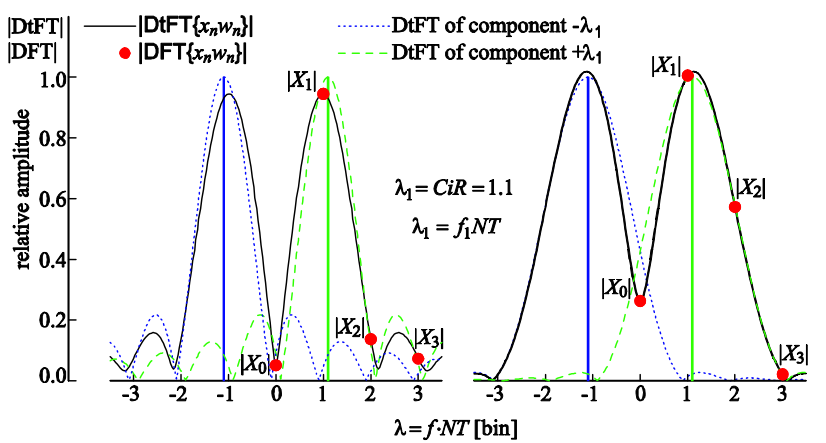

Fig. 1. The DtFT spectrum and its DFT samples of sinusoidal signal as a sum of two components; example for a) rectangular window and b) Hanning window for $\mathrm{CiR}=1.1$.

\section{Algorithm for any $H$}

When $\lambda \in\left[-\lambda_{\mathrm{x}}, \lambda_{\mathrm{x}}\right]$ where $\lambda_{\mathrm{x}}$ stands for the range of $\lambda$ for which the effect of other signal components is negligible, based on (5) and accounting for two 
components, with a frequency $\lambda_{1}$ and $-\lambda_{1}$, the Fourier transform is:

$$
X(\lambda)=\frac{D\left(\lambda+\lambda_{1}\right)}{P\left(\lambda+\lambda_{1}\right)}+\frac{D\left(\lambda-\lambda_{1}\right)}{P\left(\lambda-\lambda_{1}\right)}
$$

Transforming (12) allows to obtain generalized equations for any $H$ :

where

$$
\Pi_{1}-\lambda_{1}^{2} \Pi_{2}=0
$$

$$
\begin{gathered}
\Pi_{1}=\left|\begin{array}{ccc}
(2 H-1) H & (2 H-1) & X_{k-1}-X_{k} \\
-k^{2}-H^{2} & 2 k & X_{k} \\
(2 H-1) H & -(2 H-1) & X_{k+1}-X_{k}
\end{array}\right| \\
\Pi_{2}=\left|\begin{array}{ccc}
1 & -(2 H-1) & X_{k-1} \\
1 & 0 & X_{k} \\
1 & (2 H-1) & X_{k+1}
\end{array}\right|
\end{gathered}
$$

\section{Simulation research}

To confirm the proper functioning of this algorithm, special simulation studies in Matlab were performed.

In the first phase, the systematic error for a pure sinusoidal signal without noise versus the number of samples $N, k$ parameter and the width of the measurement window $C i R$ for $H=3$ was examined. The phase angle $\varphi_{1}$ was changed from 0 to $2 \pi$ rad every $0.01 \mathrm{rad}$. The tests were performed for different values of $N$, which were the powers of $2(32, \ldots, 256)$ because of using the FFT radix-2 algorithm.

The relative error $\left|\delta f_{1}\right|$ for the worst phase angle case of the proposed method is inversely proportional to $N^{4}$. The increase in the number of samples $N$ (at a constant $C i R$ value) decreases the value of the systematic error (Fig. 2). For $H=3$ the minimum of $\left|\delta f_{1}\right|$ is obtained for $k=1$ and $C i R$ below approx. 2.15, $k=2$ and $C i R$ above approx. 2.15. For various $H$ values this limit is different: for $H=2$ - approx. 2.08, for $H=4$ - approx. 2.29, for $H=5$ - approx. 2.49, for $H=6$ - approx. 2.86, for $H=7$ approx. 3.35 (simulations include $C i R$ values only up to 3.0).

In the second phase, the accuracy of the estimation of the signal frequency in the presence of white noise was tested for different $H$ values, to determine the statistical properties of the method with respect to the Cramer-Rao bound $(C R B)$, which sets the lower limit of the estimator's variances for the optimal method. The variance estimator was determined by calculating the $e M S E$ error - Empirical Mean Square Error. The number of instantiation was $10^{6}$ and the $C i R, k$ and $N$ values were $1.9,1$ and 512 respectively. The values of $H$ were changed from 2 to 7 .

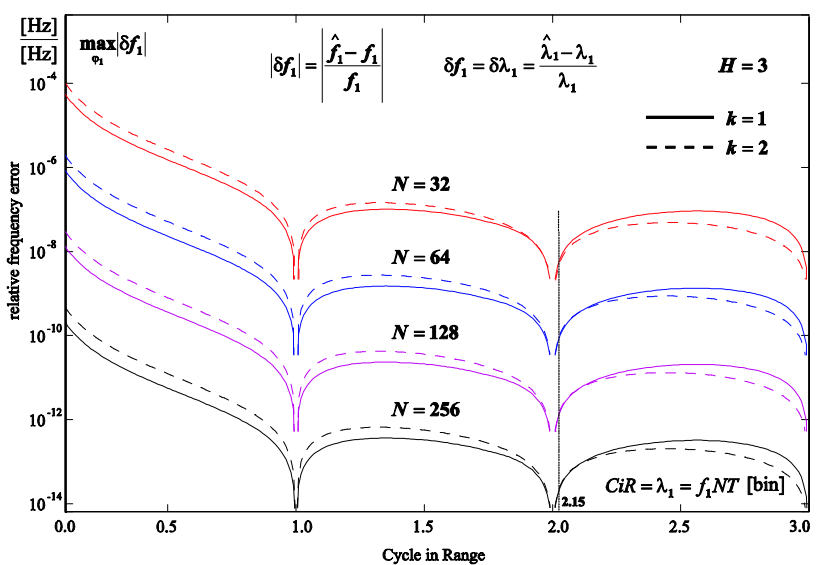

Fig. 2. The systematic error $\delta f_{1}$ of the $f_{1}$ estimation for the method of Section 3 for $H=3$ and various $k$ : $\left|\delta f_{1}\right|$ is inversely proportional to $N^{4}$, and $\delta f_{1}$ minimum is for $k=1$ for $C i R$ below approx. 2.15 and $k=2$ for $C i R$ above approx. 2.15.

Maximum decay sidelobes windows are characterized by parameters such as: MLBW (Main Lobe Band Width), ENBW (Equivalent Noise Band Width), and decay of sidelobes (in $\mathrm{dB} / \mathrm{oct}$ ). The values of these parameters increase as the $H$ value increases. The wider the main lobe, the lower frequency resolution of the window. The higher the ENBW value, the greater the influence of the noise on the spectral analysis of the measured signal. The faster the decay of the sidelobes, the better the component separation in the signal spectrum. This is especially important if the components have small amplitudes.

The increase in the value of $H$ increases the value of the frequency error as a function of $S N R$ value (Fig. 3). The $f_{1}$ frequency with reference to the Cramer-Rao bound (the quotient of the values) is approximately 1.8 for $H=2$ and approximately 4.5 for $H=7$ (Fig. 4).

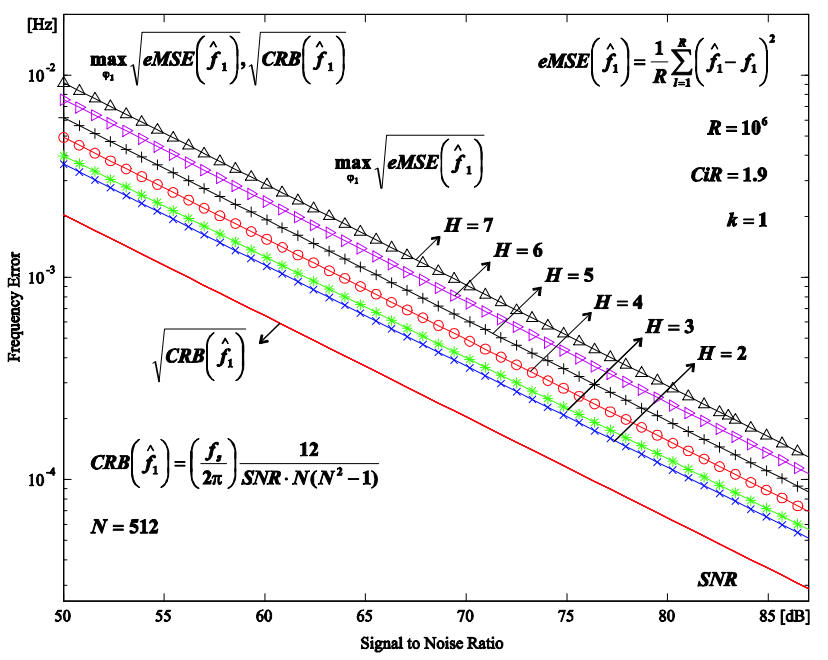

Fig. 3. Statistical properties of the proposed method for estimating the $f_{1}$ frequency: the root-squared $e M S E$ (taken as the error) method, (standard deviation) and the reference to the $C R B$ Cramer-Rao bound as a function of $S N R$ and for $C i R=1.9, k=1$ and six $H$ values in the measurement window. 


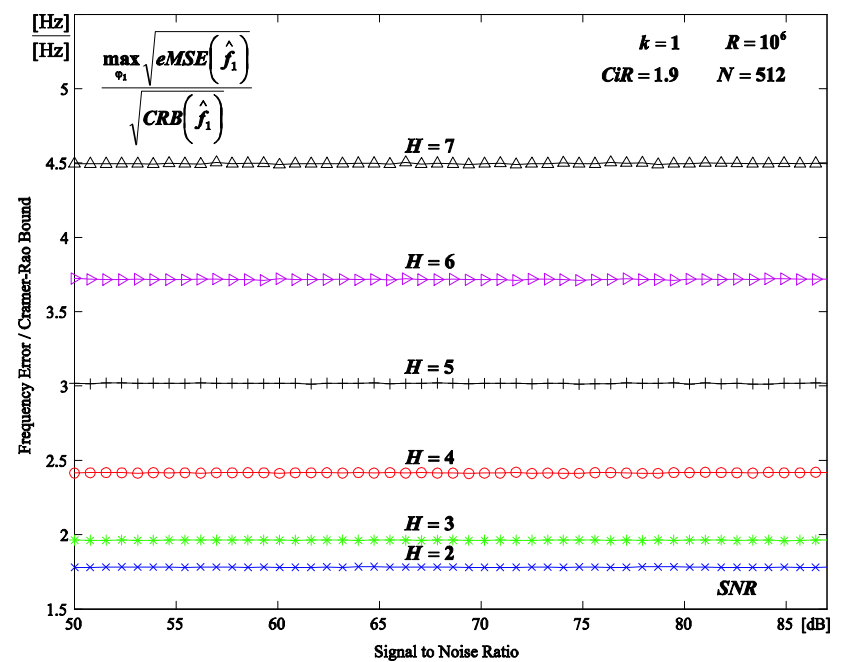

Fig. 4. Statistical properties of the proposed method for estimating the $f_{1}$ frequency with reference to the Cramer-Rao bound: the quotient of the values from figure 3 .

\section{Conclusions}

This article presents a general formula that is used to estimate the frequency of sinusoidal signal using the Fast Fourier Transform and maximum decay sidelobes windows for the values of $1<H \leq 7$. Using different windows from the discussed family allows to adjust to the actual requirements of the estimation to the appropriate applications.

Digital simulations confirm the correctness of the formula (13) for any $H$. The ratio of the estimated $f_{1}$ standard deviation (eMSE square root in the case of an unbiased estimator) to the minimum variance estimator standard deviation (CRB bound) remains constant within specified limits, regardless of the SNR (Signal to Noise Ratio) ratio value for typical tested $S N R$ values. The increase in the value of $H$ increases the value of the frequency error as a function of $S N R$ value. The estimated frequency with reference to the Cramer-Rao bound (the quotient of the values) is approximately 1.8 for $H=2$ and approximately 4.5 for $H=7$. The lower the $H$ value, the closer the curve of variation is to the $C R B$ bound. In comparison with the other spectral methods, this value is from approximately 2.14 to approximately 11.24 times lower [34]. The error values and the $C R B$ bound decrease together with the increase in the $S N R$ ratio.

The value of the relative error of the signal frequency estimation without the presence of noise depends on the $N$, $k, C i R$ and $H$ values. The value of this error is constant and amounts to approximately $10^{-12}$ when the $C i R$ value is equal to $1.5, H$ value equal to 2 and when $N=256$. For the $5 \mathrm{~ms}$ measurement window, the value of the relative error is constant and amounts to approximately $5 \cdot 10^{-11}$. In comparison, the relative errors of the other spectral methods amount to approximately $10^{-7} \mathrm{~Hz}$ [34]

The systematic error for the worst phase angle case of the proposed method is inversely proportional to $N^{4}$. The increase in the number of samples $N$ (at a constant CiR value) results in the decrease of the systematic error value. The increase in the $H$ value (at a constant $C i R$ value) results in the increase of the systematic error value.
The simulation results show that the presented algorithm can be successfully applied to frequency estimation of the grid signal e.g. in a renewable system and power control system. The new method can contribute to the improvement of the quality of produced energy and increase the efficiency of the entire power system. The possibility of using different windows from the discussed family has some advantages - it is possible to match the specific window data to the actual requirements of electricity generation. In addition, the discussed estimation method applies to one sinusoid, but it can be generalized to a multi-frequency signal. The implementation of the algorithm is straightforward and doesn't take much time.

\section{References}

[1] N.A. Rahim, J. Selvaraj, K.H. Solangi, "Energy policy to promote photovoltaic generation", Renewable and Sustainable Energy Reviews, Vol. 25, pp. 44-58, 2013.

[2] K. Kelley, R.P. Light, R. Agarwal, "Trended cosinor change model for analyzing hemodynamic rhythm patterns in hemodialysis patients", Hypertension, No. 50, pp. 143150, 2007.

[3] J.J. Blaira, T.E. Linnenbrinkb, "Corrected rms error and effective number of bits for sine wave ADC tests", Computer Standards and Interfaces, No. 26, pp. 43-49, 2003.

[4] R. Targosz, J. Manson, "Pan European LPQI Power Quality Survey", 19th International Conference on Electricity Distribution, Vienna, 2007.

[5] R. Teodorescu, F. Blaabjerg, U. Borup, M. Liserre, "A New Control Structure for Grid-Connected LCL PV Inverters with Zero Steady-State Error and Selective Harmonic Compensation", Nineteenth Annual IEEE Applied Power Electronics Conference and Exposition, pp. 580-586, 2004.

[6] J.-W. Choi, Y.-K. Kim, H.-G. Kim, "Digital PLL control for single-phase photovoltaic system", IEEE Proc.-Electr. Power Appl., Vol. 153, No. 1, 2006.

[7] R. Agha, "Performance control of distributed generation using digital estimation of signal parameters", Queensland University of Technology, 2010.

[8] G. Fedele, C. Picardi, D. Sgrò, "An estimation and synchronization method based on a new modeling approach of power electrical signals", Conference on Renewable Energies and Power Quality Valencia, 2009.

[9] M.A.G. Brito, L.P. Sampaio, G. Jr. Luigi, A. Guilherme, C. A. Canesin, "Comparative Analysis of MPPT Techniques for PV Applications", International Conference on Clean Electrical Power, pp. 99-104, 2011.

[10] A. Barchowsky, J. P. Parvin, G.F. Reed, M. J. Korytowski, B. M. Grainger, "A Comparative Study of MPPT Methods for Distributed Photovoltaic Generation", IEEE PES Innovative Smart Grid Technologies, pp. 1-7, 2011.

[11] J. Selvaraj, N. A. Rahim, C. Krismadinata, "Digital PI Current Control for Grid Connected PV Inverter", 3rd IEEE Conference on Industrial Electronics and Applications, pp. 742-746, 2008.

[12] H. Cha, T. Vu, J. Kim, "Design and Control of Proportional-Resonant Controller Based Photovoltaic Power Conditioning System", IEEE Energy Conversion Congress and Exposition, pp. 2198-2205, 2009.

[13] M. Ciobotaru, "Reliable Grid Condition Detection and Control of Single-Phase Distributed Power", Faculty of Engineering, Aalborg University, 2009. 
[14] J. A. Suul, "Control of Grid Integrated Voltage Source Converters under Unbalanced Conditions", doctroral thesis, 2012.

[15] R. G. Lyons, "Understanding Digital Signal Processing", Prentice Hall, 2004.

[16] A. V. Oppenheim, R. W. Schafer, "Digital Signal Processing", Englewood Cliffs, Prentice-Hall, 1975.

[17] A. V. Oppenheim, A. S. Willsky, S. H. Nawab, "Signals \& Systems", Prentice Hall, 1997.

[18] M. Sedlacek, M. Titera, "Interpolations in frequency and time domains used in FFT spectrum analysis", Measurement, Vol. 23, pp. 185-193, 1998.

[19] D. Agrež, "Weighted Multipoint Interpolated DFT to Improve Amplitude Estimation of Multifrequency Signal", IEEE Transactions on Instrumentation and Measurement, Vol. 51, No. 2, pp. 287-292, 2002.

[20] D. Belega, D. Dallet, "Frequency estimation via weighted multipoint interpolated DFT", Measurement and Technology, Vol. 2, No. 1, pp. 1-8, 2008.

[21] Y. F. Li, K. F. Chen, "Eliminating the picket fence effect of the fast Fourier transform", Computer Physics Communications, Vol. 178, pp. 486-491, 2008.

[22] K. F. Chen, S. L. Mei, "Composite Interpolated Fast Fourier Transform With the Hanning Window", IEEE Transactions on Instrumentation and Measurement, Vol. 59, No. 6, pp. 1571-1579, 2010.

[23] D. Belega, D. Dallet, D. Petri, "Statistical description of the sine-wave frequency estimator provided by the interpolated DFT method", Measurement, Vol. 45, No. 1, pp. 109-117, 2012.

[24] K. F. Chen, J. T. Jiang, S. Crowsen, "Against the long-range spectral leakage of the cosine window family", Computer Physics Communications, Vol. 180, pp. 904-911, 2009.

[25] J. Borkowski, "Continuous and discontinuous linear approximation of the window spectrum by least squares method", Metrol. Meas. Syst., Vol. 18, No. 3, pp. 379-390, 2011.

[26] J. Borkowski, "LIDFT - the DFT linear interpolation method", IEEE Transactions on Instrumentation and Measurement, Vol. 49, pp. 741-745, 2000.

[27] J. Borkowski, J. Mroczka, "Metrological analysis of the LIDFT method", IEEE Transactions on Instrumentation and Measurement, Vol. 51, pp. 66-71, 2002.

[28] K. F. Chen, X.Cao, Y. F.Li, "Sine wave fitting to short records initialized with the frequency retrieved from Hanning windowed FFT spectrum", Measurement, Vol. 42, No. 1, pp. 127-135, 2009.

[29] D. C. Rife, G.A. Vincent, "Use of the Discrete Fourier Transform in the Measurement of Frequencies and Levels of Tones", Bell System Technical Journal, Vol. 49, pp. 197228. 1970.

[30] D. Belega, D. Dallet, "Multifrequency signal analysis by Interpolated DFT method with maximum sidelobe decay windows", Measurement, Vol. 42, pp. 420-426, 2009.

[31] D. Belega, "The maximum sidelobe decay windows", L'Académie Roumaine, Revue Roumaine des Sciences Techniques. Série Electrotechnique et Energetique, Vol. 3, pp. 349-356, 2005.

[32] D. Agrez, "Dynamics of Frequency Estimation in the Frequency Domain", IEEE Transactions on Instrumentation and Measurement, Vol. 56, No. 6, pp. 2111-2118, 2007.

[33] D. Belega, D. Dallet, D. Petri, "Uncertainty Analysis of the Normalized Frequency Estimation by Multipoint Interpolated DFT Approach", The International Workshop on Advanced Methods for Uncertainty Estimation in Measurement, Bucharest, pp. 34-38, 2009.

[34] T. P. Zieliński, K. Duda, "Frequency and damping estimation methods - an overview", Metrol. Meas. Syst., Vol. 18, No. 4, pp. 505-528, 2011.

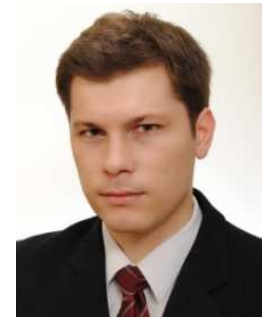

Dariusz Kania received B.S. and M.S. degrees in electronics from Wrocław University of Technology, Poland in 2011. He is currently pursuing a Ph.D. degree in electronics at Wrocław University of Technology and Chair of Electronic and Photonic Metrology.

His research interests include the development of methods and algorithms for signal processing and the use of renewable energy sources (especially photovoltaic panels) to produce electrical power. He analyzes experimental and simulation data mainly in Matlab/Simulink and implements algorithms on signal processors.

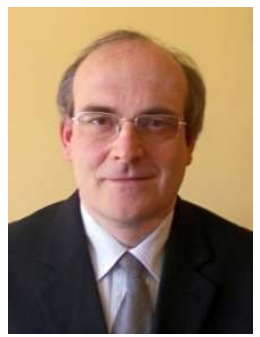

Józef Borkowski received an M.Sc. degree from the Electronic Department at Wroclaw University of Technology, Wroclaw, Poland in 1986. He received a Ph.D. degree in 1997 for his dissertation "The DFT linear interpolation method in the analysis of measuring sensor signal", and a D.Sc. degree in 2012.He is currently an Associate Professor at Wroclaw University of Technology, the Chair of Electronic and Photonic Metrology. His main research areas are: measurement of data processing using digital signal processing methods, algorithms, hardware platforms and software; methods of time-frequency transformations; precise spectrum analysis methods (methods of linear interpolation of discrete Fourier transform); image analysis methods (geometric matching of circular elements); and methods of analysis and data processing in renewable energy systems.

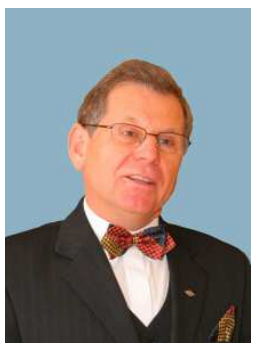

Janusz Mroczka is a professor of electronic and photonic metrology at Wroclaw University of Technology. He was born in Poland in 1952. In 1991, he received the higher doctor's degree (habilitation) for a thesis in metrological problems of scattered light application on particle size distribution investigation in dispersed solutions, from the same university. Since 1998, he has been the head of the Chair of Electronic and Photonic Metrology at Wroclaw University of Technology.

His research interests are concentrated on physical and mathematical modeling in complex measurements, inverse problem, intelligent measuring devices, scattering radiation spectral and polarization analysis, and multisensor data fusion and time-frequency representations in data processing.

Professor Mroczka is a member of SPIE, a member of the Polish Physical Society, and President of the Committee for Metrology and Instrumentation at the Polish Academy of Science. Since 2010, he has been a Corresponding Member of the Polish Academy of Science. 